\title{
ASPECTOS CLÍNICOS E RADIOGRÁFICOS DA OSTEODISTROFIA HIPERTRÓICA EM CÃES. ESTUDO RETROSPECTIVO DE 16 CASOS
}

\author{
CLINICAL AND RADIOGRAPHIC ASPECTS OF HYPERTROPHIC OSTEODYSTROPHY IN THE \\ DOGS. A RETROSPECTIVE STUDY OF SIXTEEN CASES
}

\author{
Julio Carlos Canola ${ }^{1} \quad$ Alexandre Lima de Andrade ${ }^{2}$
}

\section{RESUMO}

Descrevem-se 16 casos de osteodistrofia hipertrófica (OH) entre 13.483 casos levantados nos arquivos do Setor de radiologia do Hospital Veterinário "Governador Laudo Natel" da FCAVJ - UNESP, entre $l^{\circ}$ de janeiro de 1977 a 31 de dezembro de 1995. A enfermidade mostrou-se de baixa manifestação clínica, atingindo cães jovens em fase de crescimento, pertencentes às raças de grande porte e supernutridos. Destaca-se ainda, a importância do exame radiográfico seriado no diagnóstico da enfermidade e principalmente como método de avaliação da reversão clínica do processo, mormente quando ocorre bacteremia associada.

Palavras-chaves: osteodistrofia hipertrófica, bacteremia, cão

\section{SUMMARY}

Sixteen cases of hypertrophic osteodistrophy (HOD) among 13.483 cases surveyed from the files of the Radiology Sector of Veterinary Hospital "Governador Laudo Natel" of FCAVJ UNESP, between January 1st, 1977 and December 31st, 1995, were described. The illness showed a low clinical manifestation, reaching large-breeded young dogs at growing phase, and fed with a nutritional oversupplementation. It is still highlighted, the importance of the serial radiographic exam on the disease diagnosis, and mainly as an assessment method of the clinical process reversion, specially when an associated bacteraemia takes place.

Key words: hypertrophic osteodistrophy, bacteraemia, dog.

\section{INTRODUÇÃO:}

A osteodistrofia hipertrófica $(\mathrm{OH})$ é também encontrada na literatura com o nome de escorbuto canino, escorbuto ósseo, doença de Moler-Barlow, doença de Barlow, osteopatia metafisária, osteodistrofias I e II.

Esta síndrome foi inicialmente descrita por Garlic apud IAMAGUTI et al. (1988) no ano de 1946, sob a denominação de osteodistrofia hipertrófica. Mais tarde, HOLMES (1962), MEIER et al. (1967), OLSSON (1972) e WATSON \& FARROW (1973) descreveram as alterações encontradas, corroborando a denominação proposta anteriormente. Entretanto, Putman \& Archibald (1968) apud SANTOS \& HAGIWARA (1992) referiram-se às mesmas alterações com o nome de escorbuto canino. Foi criada grande polêmica em 1972 quando Buyere apud SANTOS \& HAGIWARA (1992), mencionou que se tratavam de doenças diferentes. Tal fato foi, no entanto, contestado por BENNETT (1976), que afirmou tratar-se da mesma doença, diferentes apenas quanto à evolução $\mathrm{e}$ ao grau de severidade.

A doença acomete normalmente cães jovens, em fase de crescimento, pertencentes às raças de grande porte ou gigantes (BENNETT, 1976;

${ }^{1}$ Médico Veterinário, Doutor, Professor Assistente, Departamento de Clínica e Cirurgia Veterinária (DCCV), Faculdade de Ciências Agrárias e Veterinárias (FCAV), Campus de Jaboticabal, UNESP, Rodovia Carlos Tonanni, km 05, 14870-000 Jaboticabal, SP. Autor para correspondência.

Médico Veterinário, Pós-graduando em Medicina Veterinária, Área de Concentração em Cirurgia Veterinária, FCAV, UNESP, Campus de Jaboticabal. 
DOIGE, 1990) e de rápido crescimento (LENEHAN \& FETTER, 1985). A idade de maior ocorrência é a de três a sete meses (BENNETT, 1976; GRONDALEN, 1976; Alexander apud HOSKINS, 1992) e, quanto ao sexo, os machos são mais freqüentemente afetados do que as fêmeas (GRONDALEN, 1976).

A etiologia da osteodistrofia hipertrófica é incerta, mas a superalimentação, agentes infecciosos e a hipovitaminose $C$ têm sido sugeridas como causas (GRONDALEN, 1976; TEARE $\boldsymbol{e}$ t al., 1979). A suplementação vitamínica e mineral em excesso na dieta pode ser a causa da doença. Isto foi sugerido por TEARE et al. (1979), quando observaram que cães das raças Dogue Alemão e Labrador Retrieviers, alimentados com dieta de alta palatabilidade e à vontade, desenvolveram alterações esqueléticas. De acordo com estes autores, as alterações podem ter sido ocasionadas por hipercalcitonemia, decorrente de uma diminuição da reabsorção óssea induzida pela dieta rica em cálcio, proteína e energia.

WATSON \& FARROW (1973) referiramse ao aumento da concentração sérica de fósforo em animais com OH. Por sua vez, MERINO et al. (1988), ao estudarem 18 cães jovens das raças Pastor Alemão e Dobermann submetidos a uma dieta rica em minerais, especialmente em fósforo, concluíram que este elemento em excesso é a causa provável da doença. Segundo estes autores, a acidose metabólica observada seria responsável pela inibição da atividade osteoblástica.

A deficiência de vitamina $C$ foi também sugerida como causa, devido à semelhança da doença com o escorbuto viste em crianças (BENNETT, 1976). MEIER et al. (1967) relataram sete casos de $\mathrm{OH}$, em cães, associando-os à hipovitaminose $\mathrm{C}$. Mais tarde, BENNETT (1976) admitiu ser incerto que a deficiência de vitamina $C$ possa ser etiologicamente importante. GRONDALEN (1976), estudando 26 cães afetados pela doença, concluiu que existem outros fatores envolvidos, além da deficiência de vitamina $C$.

$\mathrm{O}$ excesso de vitamina $\mathrm{D}$ poderia ser $\mathrm{o}$ fator primordial, uma vez que já foi encontrado, em um caso da doença, calcificação de tecidos moles e de vasos (BENNETT, 1976).

A sugestão de uma etiologia infecciosa não é substancial. A tentativa de transmissão da doença por transfusão sangüínea, não apresentou resultados positivos. No entanto, SCHULZ et al. (1991) relataram um caso da doença associada à bacteremia por Escherichia coli. De acordo com os autores, as infecções sistêmicas podem ser causas contribuintes da $\mathrm{OH}$. Porém, agentes infecciosos não foram isolados das lesões ósseas (WOODWARD, 1982; SCHULZ et al., 1991). Provavelmente a bacteremia desenvolvida é secundária ao estresse e à anorexia, freqüentemente associada à doença.

Os cães apresentam, clinicamente, tumefações dolorosas, de grau leve a moderado, principalmente nas metáfises distais do rádio e da ulna. Claudicação, andar relutante, febre, letargia e anorexia estão freqüentemente presentes (GRONDALEN, 1976). A doença pode regredir espontaneamente ou progredir para formas mais graves. Nesta fase, o animal pode desenvolver alterações metabólicas, desequilíbrio ácido-básico e alterações nos níveis de glicose secundariamente à anorexia e à hipertermia prolongada, que podem provocar a morte do animal (GRONDALEN, 1976; NEWTON \& SIEMERING, 1983). Hemorragias gengivais, subcutâneas e cutâneas, anemia e diarréia hemorrágica são outros sinais clínicos relatados na literatura (Garlick apud IAMAGUTI et al., 1988).

As análises sangüíneas, referentes a hemograma e às provas bioquímicas podem estar normais ou revelarem aumento na contagem de leucócitos (NEWTON \& SIEMERING, 1983). Os níveis séricos de cálcio, fósforo, fosfatase alcalina e ácido ascórbico geralmente estão dentro da normalidade (GRONDALEN, 1976).

O diagnóstico pode ser confirmado por radiografias. $\mathrm{Na} \mathrm{OH}$ precoce são evidenciadas linhas ou faixas radiotransparentes anormais no interior das metáfises distais dos ossos longos. Estas linhas são mais evidentes na porção distal do rádio e da ulna, podendo ser observadas em outras regiões, como na tíbia e nas junções costocondrais (LENEHAN \& FETTER, 1985).

A calcificação da matriz cartilaginosa é normal formando a zona de calcificação provisória e a esponjosa primária que, no entanto, não se remodela. Desta forma, a esponjosa primária persiste e torna-se mais espessa, formando uma linha radiotransparente entre a cartilagem e a metáfise. Com o progredir da doença, forma-se "novo" osso periosteal em torno da metáfise que, às vezes, envolve toda a diáfise. A linha de crescimento e a epífise, de modo geral, não estão envolvidas. A tumefação de tecidos moles também pode ser observada radiograficamente. Conforme ocorre a evolução para a cura, na maioria dos casos, pode-se observar cicatrização óssea residual ou espessamento da metáfise adjacente à neoformação óssea. Nos casos mais graves e crônicos, a remodelação periosteal é anormalmente intenso, resultando em deformidades do membro (HOLMES, 1962; OLSSON, 1972; LENEHAN \& FETTER, 1985; IAMAGUTI et al., 1988; SCHULZ et al., 1991). 
Microscopicamente foram evidenciadas ampliação da esponja primária, necrose de osteoblastos, ausência de deposição de osteóide nos núcleos cartilaginosos calcificados, microfraturas trabeculares e infiltração de neutrófilos entre as trabéculas da esponja primária (SANTOS \& HAGIWARA, 1992).

Inúmeros tratamentos têm sido propostos, entretanto, não oferecem resultados consistentes. $\mathrm{O}$ tratamento deve ser direcionado para o controle da febre e redução da dor. Repouso e analgésicos são geralmente suficientes, sendo os corticóides reservados apenas para casos mais graves (LENEHAN \& FETTER, 1985).

BENNETT (1976) relatou que, em alguns casos, a administração da vitamina $\mathrm{C}$ proporciona completa remissão dos sinais clínicos. Todavia, TEARE et al. (1979) demonstraram, experimentalmente, que essa terapia não beneficiava, podendo muitas vezes agravar as condições clínicas dos pacientes.

A correção da dieta, retirando-se o excesso de proteína, vitaminas, minerais e energia (MERINO et al., 1988; SANTOS \& HAGIWARA, 1992), associada à administração de ácido acetil salicílico para o controle de dor (MERINO et al., 1988), tem sido o tratamento mais eficaz.
A maioria dos cães exibe remissão espontânea da doença, à despeito da modalidade terapêutica usada, dentro de dias ou semanas. Em casos graves, o animal pode ficar com deformidades ósseas permanentes ou sucumbir à moléstia, devido a hipertermia. As recidivas são raras (LENEHAN \& FETTER, 1985). Tem sido sugerido que os casos fatais são decorrentes da liberação de toxinas dos focos necróticos, do tecido ósseo (SANTOS \& HAGIWARA, 1992).

\section{MATERIAIS E MÉTODOS}

\section{Material Biológico}

Descrevem-se 16 casos entre 13.843 atendiáos junto ao Setor de Radiologia do Hospital Veterinário "Governador Laudo Natel" da FCAV UNESP (Faculdade de Ciências Agrárias e Veterinárias), no período de $1^{\circ}$ de janeiro de 1977 a 31 de dezembro de 1995. Os 16 animais, 10 machos e 6 fêmeas, perterciam à espécie canina, de raças variadas e com idade compreendida entre 3 e 6 meses (Quadro $1)$.

\begin{tabular}{|c|c|c|c|c|}
\hline Caso & Raça & Idade & Sexo & Membro afetado \\
\hline 01 & SRD & 3 meses & macho & MT bi / MP bi \\
\hline 02 & Dogue Alemão & 6 meses & macho & MT bi / MP bi \\
\hline 03 & Dogue Alemão & 5 meses & macho & MT bi \\
\hline 04 & Dogue Alemão & 6 meses & macho & MT bi \\
\hline 05 & Boxer & 4 meses & macho & MT bi / MP bi \\
\hline 06 & Boxer & 3 meses & macho & MT bi \\
\hline 07 & Pastor Alemão & 3 meses & fêmea & MT bi \\
\hline 08 & Dogue Alemão & 4 meses & fêmea & $\overline{M T P}$ bi \\
\hline 09 & Boxer & 4 meses & fêmea & MT bi \\
\hline$\overline{10}$ & Dogue Alemão & 4 meses & fêmea & MT bi \\
\hline 11 & Dogue Alemão & 4 meses & macho & $\mathrm{MP}$ bi \\
\hline 12 & Weimaraner & 3 meses & fềmea & MT bi \\
\hline 13 & Boxer & 3 meses & macho & MT bi \\
\hline$\overline{14}$ & Weimaraner & 4 meses & fêmea & MT bi \\
\hline$\overline{15}$ & Rottweiler & 6 meses & macho & MT bi \\
\hline 16 & Weimaraner & 4 meses & macho & $\mathrm{MT}$ bi / AC / MP bi \\
\hline
\end{tabular}

MT: membro torácico; MP: membro pélvico; AC: articulação costocondral; bi: bilateral. 


\section{História Clínica}

Animais de criação doméstica, alimentados com ração comercial para filhotes, carne bovina, ovos, frutas, farinha de milho, leite, vísceras de animais e suplementação vitamínica e mineral, todos oferecidos ad libitum. Os animais atendidos manifestavam febre e relutância para se moverem e cinco deles apresentavam anorexia e prostração; todos com evolução clínica de aproximadamente 20 dias na apresentação.

\section{Semiotécnica}

Frente a queixa, os animais foram submetidos ao exame físico de rotina, no qual foram analisados, na ordem de avaliação, temperatura corporal, mucosas aparentes, linfonodos, freqüência cardíaca e respiratória, palpação abdominal e dos membros torácicos e pélvicos.

Com base nestes achados, delineou-se utilizar métodos diagnósticos complementares como hemograma, técnicas radiográficas simples (abordagem pelas incidências lateral e anteroposterior), principalmente dos membros torácicos.

\section{Terapêutica}

Nortearam-se as medidas de forma a aliviar a dor e a pirexia, bem como a resolução do processo. Os animais foram submetidos a terapia com vitamina $\mathrm{C}^{\mathrm{a}}$ (2 gramas diárias VO) e dipirona ${ }^{\mathrm{b}}$ (uma gota por quilograma de peso, VO, TID). Foi orientada a retirada de toda suplementação vitamínica e mineral, bem como a correção da dieta. Nos referidos casos de bacteremia associada (Figs. 1, 2 e 3), foi preconizado o uso de ampicilina benzatina ${ }^{c}$ (50 $\mathrm{mg} / \mathrm{kg} /$ dia, IM) durante 15 dias.

\section{RESULTADOS E DISCUSSÃO}

Dos dados obtidos, ressaltaram-se, febre (temperatura média de $40^{\circ} \mathrm{C}$ ); em dois animais as mucosas apresentavam-se congestas; em um dos casos sensibilidade em região topográfica correspondente à área renal.

Em todos os animais foram observados sinais de alargamento bilateral da metáfise distal do rádio, com sensibilidade acentuada à palpação da mesma região.

Aos resultados amontaram-se, imagem radiográfica da metáfise distal dos ossos longos, demonstrando zona radiotransparente adjacente à linha de crescimento, esclerose, bainha de calcificação envolvendo a região metafisária, normalmente separada da córtex, caracterizando em todos os casos a osteodistrofia hipertrófica.

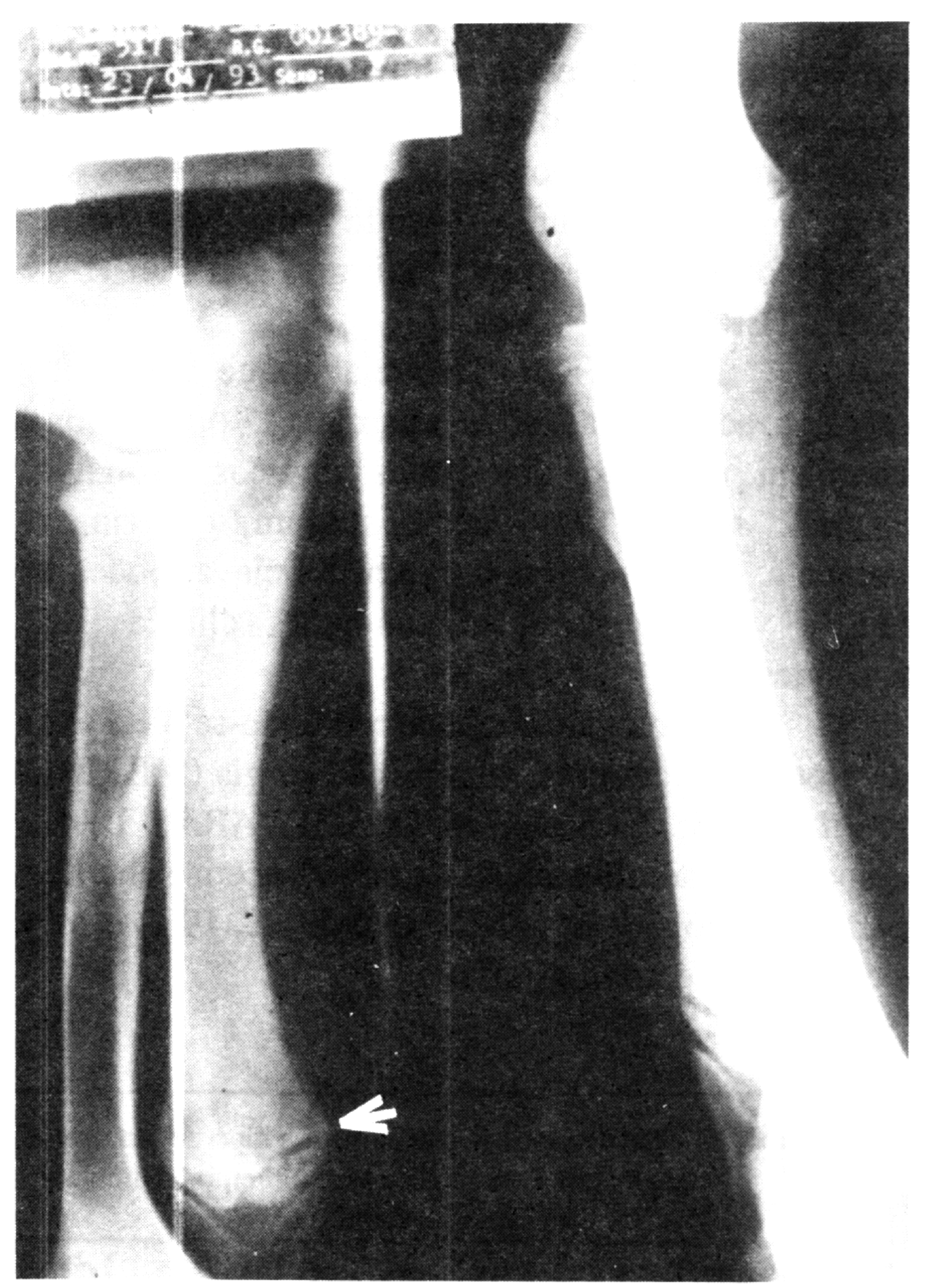

Figura 1. Aspecto radiográfico do rádio-ulna de cão Dogue Alemão, 4 meses, fềmea, apresentando $\mathrm{OH}$ associada à bacteremia por Streptococcus $\alpha$-hemolíticum (fase inicial). Notar presença de linha radiotransparente irregular proximal à placa de crescimento da metáfise distal da ulna (seta).

Em dois animais, não responsivos ao tratamento proposto, foi procedida a coleta asséptica de sangue para hemocultura e antibiograma, cujos resultados foram de Streptococcus $\alpha$-hemoliticum e Streptococcus $\beta$-hemoliticum respectivamente, com sensibilidade ao cloranfenicol $(+++)$, ampicilina $(+++)$, carbenecilina $(++)$ e norfloxacin $(+)$. Outros antibióticos foram testados, aos quais o agente mostrou resistência.

Pelo levantamento realizado, no qual procurou-se estabelecer a freqüência de diferentes patologias diagnosticadas radiograficamente junto ao 
Setor de Radiologia no período retromencionado, verificou-se que a osteodistrofia hipertrófica foi uma enfermidade de baixa manifestação clínica.

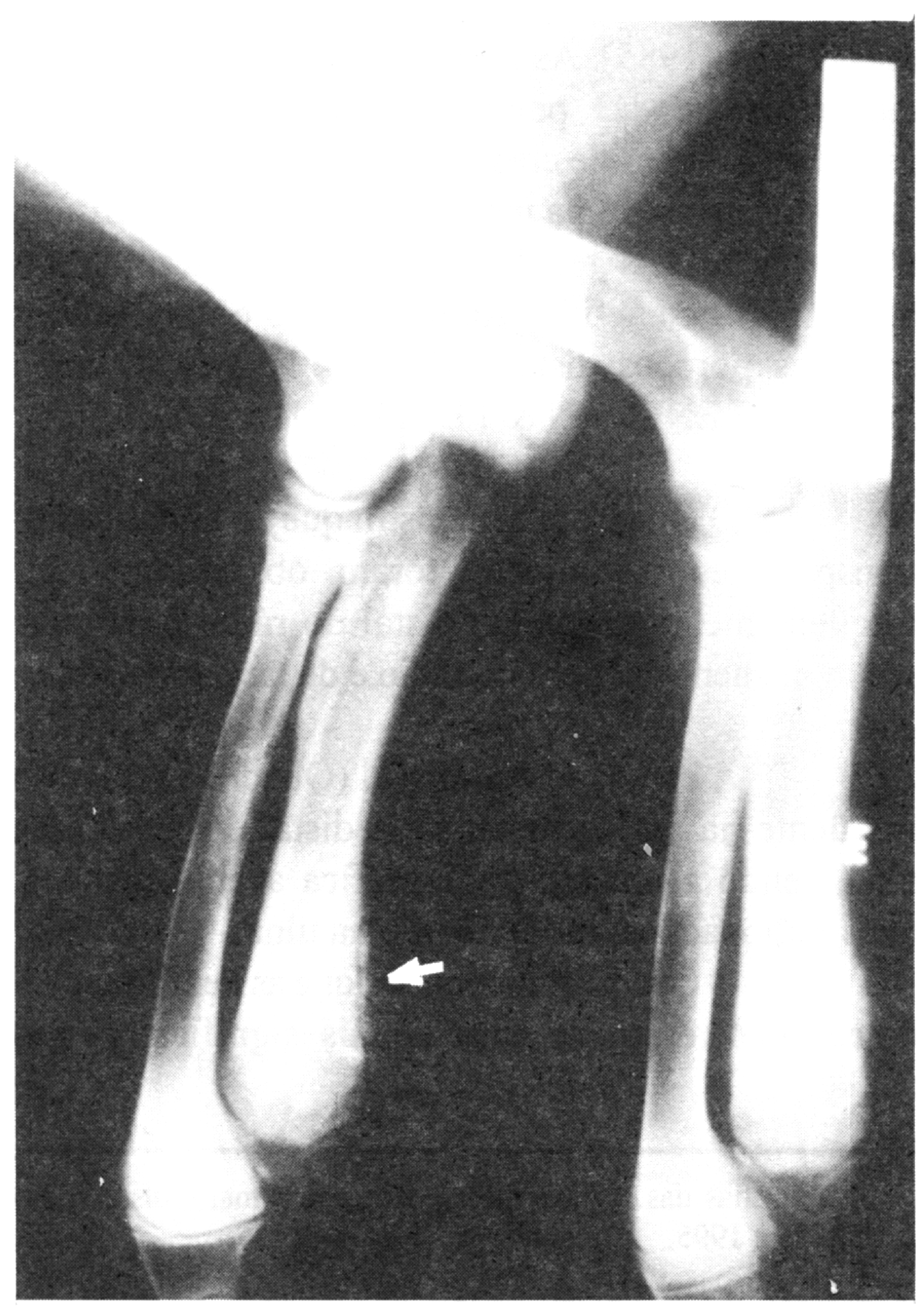

Figura 2. Aspecto radiográfico do rádio-ulna de cão Dogue Alemão. 4 meses, fềmea apresentando $\mathrm{OH}$ associada à bacteremia por Streptococcus $\boldsymbol{\alpha}$-hemoliticum (15 dias após a data de apresentação). Notar esclerose na região metafisária distal do rádio e da ulna (bilateral) e neoformação de tecido ósseo periosteal (seta).

O Quadro 1 demonstra, por ordem de entrada para o atendimento médico, o número de animais que apresentavam $\mathrm{OH}$ por raça, idade, sexo e membros acometidos.

Como citado por BENNETT (1976), LENEHAN \& FETTER (1985) e DOIGE (1990), a doença ocorreu em cães de raças grandes e gigantes. A idade de ocorrência das manifestações clínicas da enfermidade deu-se entre três e seis meses, como verificada por BENNETT (1976) e Alexander apud HOSKINS (1992). Independentemente da raça e de acordo com o descrito por GRONDALEN (1976), observou-se maior freqüência em cães do sexo masculino.
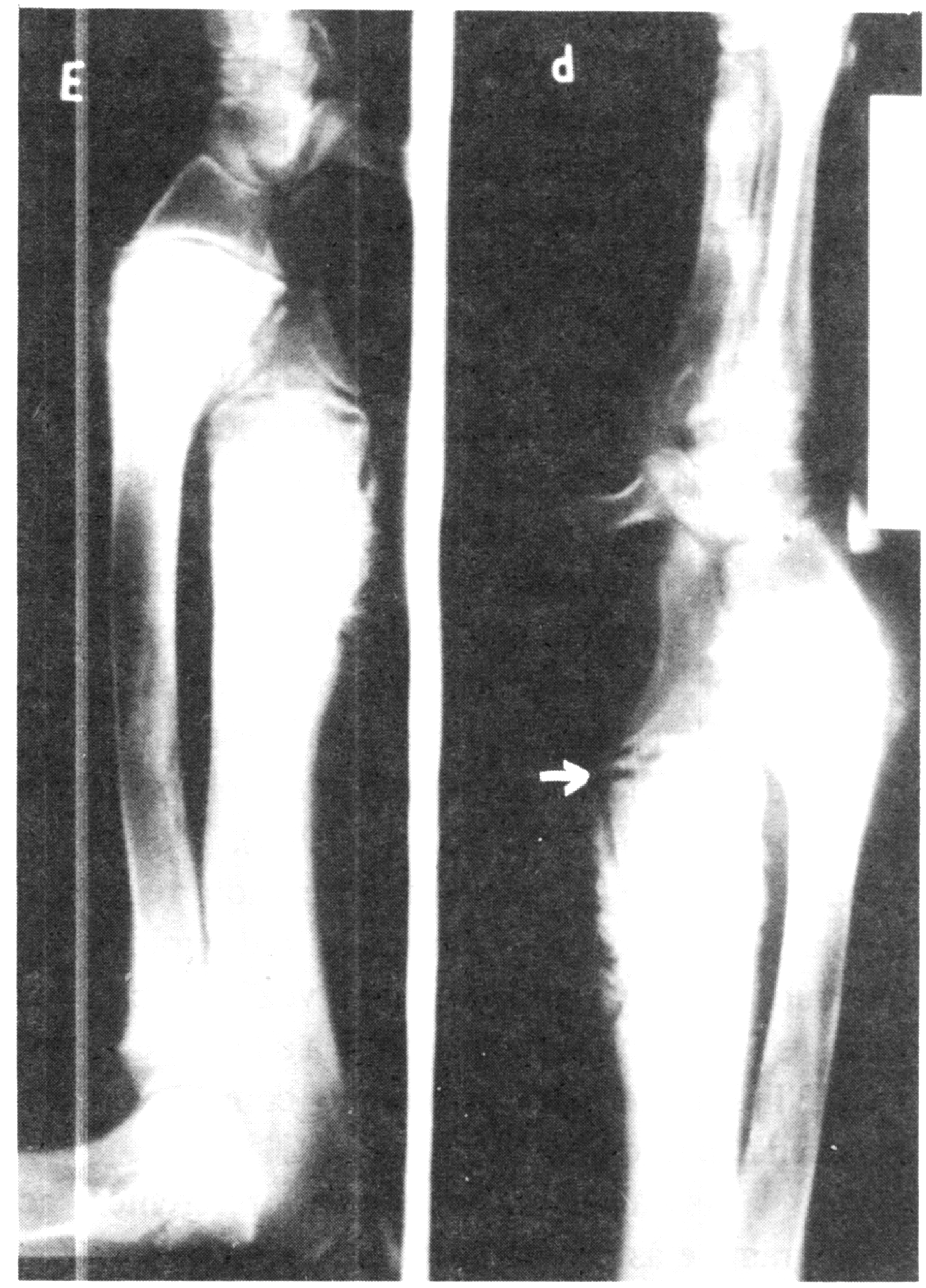

Figura 3. Aspecto radiográfico do rádio-ulna de cão Dogue Alemão, 4 meses, fềmea apresentando $\mathrm{OH}$ associada à bacteremia por Streptococcus a-hemoliticum (30 dias após a apresentação). Notar evolução da esclerose e neoformações ósseas envolvendo o rádio e ulna, reaparecimento da linha radiotransparente paralela à linha de crescimento ósseo (seta).

Mesmo diante de várias pesquisas, a etiologia da $\mathrm{OH}$ parece incerta até o presente momento. Alguns autores procuraram relacionar, sem maiores esclarecimentos, o surgimento das alterações estruturais ósseas com hipocalcitonemia (GRONDALEN, 1976; TEARE et al., 1979), elevação na concentração sérica de fósforo (WATSON \& FARROW, 1973; MERINO et al., 1988), hipovitaminose C (MEIER et al., 1957), excesso de vitamina $D$ (BENNETT, 1976) e processos infecciosos (SCHULZ et al., 1991).

Neste levantamento, o grupo de cães estudados com alterações ósseas compatíveis com $\mathrm{OH}$ eram supernutridos e recebiam suplementação vitamínica e mineral. Considerando a desequilíbrio nutricional imposto a cada animal durante a fase inicial de crescimento, pode-se admitir que a enfermidade ocorreu, em sua maioria, secundariamente à superali- 
mentação, conforme relataram BENNETT (1976) e TEARE et al. (1979). Não se pode afirmar que a $\mathrm{OH}$ foi ocasionada por excesso ou deficiência de microe lementos, uma vez que seu nível de fornecimento não foi mensurado. Embora não tenha sido realizada a necropsia dos animais para a identificação das lesões e isolamento do agente infeccioso, como o descrito por SCHULZ et al. (1991), por tratarem-se de pacientes da rotina hospitar, pressupõe-se que a ocorrência da bacteremia nos referidos casos, foi secundária ao estresse e à anorexia associada a $\mathrm{OH}$.

Os sintomas verificados, ressaltando-se a temperatura corpórea média de $40^{\circ} \mathrm{C}$, o andar relu tante, a anorexia, prostração e as tumefações doloro sas, principalmente nas regiões metafisárias distais do rádio e da ulna, foram semelhantes aos observados e descritos por GRONDALEN (1976). Durante o período de estudo, não foi observado regressão espontânea do quadro clínico e radiográfico de qualquer animal estudado, uma vez que para os mesmos foi instituída terapia clínica a base de vitami na $\mathrm{C}$, dipirona e, para os animais com bacteremia associada, ampicilina.

As análises sangüíneas referentes ao hemograma e às provas bioquímicas eram normais, em quinze dos dezesseis animais estudados, como também observaram NEWTON \& SIEMERING (1983). Na única exceção, o animal apresentava leucocitose, que foi atribuída ao quadro de bacteremia associada.

No tocante aos achados radiográficos, observou-se similitude aos descritos por LENEHAN \& FETTER (1985). As lesões mais freqüentes estiveram representadas pelas linhas radiotransparentes paralelas às linhas de crescimento ósseo, neoformação óssea subperiosteal na metáfise, esclerose metafisária e tumefação dos tecidos moles circunjacentes. Os ossos mais acometidos foram o rádio e ulna, ambos em suas porções distais (Quadro 1, 2 e 3), como relataram LENEHAN \& FETTER (1985).

O Quadro 2 demonstra que as linhas radiotransparentes patológicas foram observadas mais freqüentemente na região distal do rádio e da ulna, sendo de menor largura no rádio e de largura média na ulna.

A esclerose metafiseal (Quadro 3) foi mais freqüente na região metafisária distal do rádio e da ulna, sendo a imagem radiográfica acentuadamente mais radiopaca no rádio do que na ulna.

O Quadro 4 demonstra que as neoformações ósseas ocorreram de forma mais significativa nas

Quadro 2. Largura da linha radiotransparente patológica observada em radiografias das regioes metafisárias, proximal e distal, do rádio e da ulna de cães com osteodistrofa hipertrófica - Jaboticabal (SP), 1995.

\begin{tabular}{|c|c|c|c|c|c|c|c|c|c|c|c|c|c|c|c|c|c|}
\hline \multicolumn{2}{|c|}{ Osso } & \multicolumn{2}{|c|}{ Metáfise } & \multicolumn{14}{|c|}{ Casos } \\
\hline \multirow{3}{*}{ Rádio } & & 1 & 2 & 3 & 4 & 5 & 6 & 7 & 8 & 9 & 10 & 11 & 12 & 13 & 14 & 15 & 16 \\
\hline & Proximal & - & ++ & - & + & +++ & + & - & + & - & ++ & + & $=$ & + & + & - & ++ \\
\hline & Distal & ++ & ++ & ++ & + & +++ & ++ & ++ & + & ++ & +++ & + & + & + & + & + & +++ \\
\hline \multirow[t]{2}{*}{ Ulna } & Proximal & - & - & - & - & - & - & - & - & - & - & - & - & - & - & - & - \\
\hline & Distal & $+t$ & ++ & ++ & ++ & +++ & +++ & +++ & ++ & +++ & + & + & ++ & ++ & + & ++ & +++ \\
\hline
\end{tabular}

$(+)$ : Linha fina. $\quad(++)$ : Linha média. $\quad(+++)$ : Linha larga.

Quadro 3. Grau de esclerose observado na região metafisária, proximal e distal, do rádio e da ulna de cães com osteodistrofia hipertrófica - Jaboticabal (SP), 1995

\begin{tabular}{|c|c|c|c|c|c|c|c|c|c|c|c|c|c|c|c|c|c|}
\hline Osso & Metáfise & \multicolumn{16}{|c|}{ Casos } \\
\hline \multirow{3}{*}{ Rádio } & & 1 & 2 & 3 & 4 & 5 & 6 & 7 & 8 & 9 & 10 & 11 & 12 & 13 & 14 & 15 & 16 \\
\hline & Proximal & $\bar{t}$ & +++ & $t+$ & +++ & $\overline{+++}$ & $\bar{t}$ & - & +++ & - & $+t$ & $\overline{t+}$ & + & +++ & + & - & ++ \\
\hline & Distal & ++ & +++ & +++ & +++ & +++ & + & $\overline{++}$ & +++ & ++ & +++ & ++ & + & $\overline{+++}$ & + & + & $\overline{++}$ \\
\hline \multirow[t]{2}{*}{ Ulna } & Proximal & - & - & - & $=$ & $=$ & - & - & - & - & + & ++ & - & - & - & - & - \\
\hline & Distal & ++ & +++ & $++t$ & +++ & +++ & t & + & +++ & + & - & $+t$ & 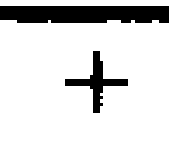 & $++t$ & + & + & + \\
\hline
\end{tabular}

$(+)$ : Grau leve. $\quad(++)$ : Grau moderado. $\quad(+++)$ : Grau intenso. 
Quadro 4. Grau de radiopacidade das neoformaçðes ósseas das regiðes metafisárias do rádio e da ulna de cães comosteodistrofia hipertrófica - Jaboticabal (SP), 1995.

\begin{tabular}{|c|c|c|c|c|c|c|c|c|c|c|c|c|c|c|c|c|c|}
\hline Osso & Epífise & \multicolumn{16}{|c|}{ Casos } \\
\hline \multirow[b]{2}{*}{ Rádio } & \multirow[b]{2}{*}{ Proximal } & 1 & 2 & 3 & 4 & 5 & 6 & 7 & 8 & 9 & 10 & 11 & 12 & 13 & 14 & 15 & 16 \\
\hline & & + & + & + & ++ & ++ & - & - & + & - & +++ & - & - & +++ & - & - & + \\
\hline \multirow[t]{2}{*}{ Ulna } & Proximal & +++ & - & ++ & - & - & - & - & - & - & + & - & - & +++ & - & - & - \\
\hline & Distal & +++ & ++ & +++ & + & $+t$ & - & - & ++ & - & + & - & - & +++ & + & - & + \\
\hline & & & & & & & & & & & & & & & & & \\
\hline
\end{tabular}

$(+)$ : Baixa intensidade; $\quad(++)$ : Média intensidade; $\quad(+++)$ : Alta intensidade.

porções proximal do rádio e distal da ulna. De modo geral, não foram encontradas lesões graves e crônicas com deformidades do crescimento como relataram HOLMES (1962) e OLSSON (1972), em virtude do diagnóstico e terapia terem sido instituídos precocemente, para a maioria dos animais.

Foi observada eficácia da vitamina $C$ na maioria dos animais, conforme relatou BENNETT (1976), confrontando com os achados experimentais de TEARE et al. (1979). O uso de analgésicos, para controle da dor e da febre, também contribuiu para o restabelecimento dos animais, conforme descreveram LENEHAN \& FETTER (1985) e MERINO et al. (1988). No entanto, acredita-se que a correção da dieta seja o passo mais importante do tratamento, uma vez que imposta a retirada do excesso de proteína, vitaminas, minerais e energia da alimentação, os animais apresentaram remissão dos sinais clínicos e melhoria no quadro radiográfico. A despeito dos animais com bacteremia associada, ressalta-se que a mesma deverá ser considerada quando há evolução do quadro clínico e radiográfico, sugerindo-se, portanto, a realização de hemocultura, antibiograma e terapia antibiótica de urgência.

\section{FONTES DE AQUISIÇÃO}

a - CEWIN - WINTROP Products Inc.

b - NOVALGINA - HOESCHST do Brasil Quím. e Farmacêutica. c - OPTACILIM 500 - BYK Química e Farcêutica Ltda.

\section{REFERÊNCIAS BIBLIOGRÁFICAS}

BENNETT, D. Nutrition and bone disease in the dog and cat. Vet Rec, v. 98, p. 310-13, 1976.

DOIGE, C. Sistema esquelético. In: THOMPSON, R. G. Patologia veterinária especial, São Paulo: Manole, 1990. cap. 11. p. 531-78.

GRONDALEN, J. Metaphyseal HOD in growing dogs: A clinical study. J Small Anim Pract, v. 17, p. 721, 1976.
HOLMES, J. R. Suspected skeletal scurvy in the dog. Vet Rec, $v$ 74, n. 29 , p. $801-13,1962$.

HOSKINS, J. D. Pediatria veterinária: cães e gatos até 6 meses de idade. São Paulo: Manole, 1992. 605 p.

IAMAGUTI, P., FERRAZ, L. M. G., GONÇALVES, C. G., et al Escobuto canino (osteodistrofia hipertrófica). Relato de um caso. Arq Flum Med Vet, v. 3, n. 4, p. 116-18, 1988.

LENEHAN, T. M., FETTER, A. W. Hypertrophic osteodystrophy In: NEWTON, C. D., NUNAMAKER, D. M. (eds): Textbook of small animal orthopaedics. Philadelphia: Lippencott, 1985. p. 597.

MEIER, H., ClARCK, S. T., SCHNELLE, G. B., et al. Hypertrophic osteodystrophy associated with disturbance of vitamin C synthesis in the dog. J Am Vet Med Assoc, v. 130, p. $11,1957$.

MERINO, N., RODRIGUEZ, E., TORRES, O., et al. Osteodistrofia hipertrófica en cachorros de las razas pastor alemán y doberman. Rvta Cub Cienc Vet, v. 19, n. 4, p. 30507,1988

NEWTON, C. D., SIEMERING, G. Skeletal diseases. In: ETTINGER, S. J. (ed): Textbook of veterinary internal medicine. Philadelphia: Saunders, 1983. p. 2236.

OLSSON, S. E. Radiology in veterinary pathology. A review with special reference to hypertrophic osteodystrophy and secundary hyperparathyroidism in the dog. Acta Radiol, Supp., v. 319. p. 225,1972

SANTOS, V. R. S., HAGIWARA, M. K. Nutrição e principais enfermidades ósseas em cães jovens. (2 parte). Cães \& Gatos, v. 43, p. 16-24, 1992.

SCHULZ. K. S., PAYNE, J. T., ARONSON, E. Escherichia coli bacteremia associated with hypertrophic osteodystrophy in a dog. J Am Vet Med Assoc, v. 199, p. 1170-73, 1991

TEARE, J. A., KROOK, L., KALLFELZ, F. A., et al. Ascorbic acid dificiency and hypertrophic osteodystrophy in the dog: a rebuttal. Cornell Vet, v. 69, p. 384-401, 1979

WATSON, B. R., FARROW, B. R. H. Hypertophic osteodystrophy in the dog. Aus Vet J., v. 49, p. 436-39, 1973.

WOODWARD, J. C. Canine hypetrophic osteodystrophy. A study of the spontaneous disease in littermates. Vet Pathol, v. 19, p. 337-54, 1982. 